\title{
Kopfschmerz durch zu viele Analgetika: ambulant entziehen oder stationär?
}

Kopfschmerz bei Medikamentenübergebrauch ist ein häufiges Problem in Kopfschmerzambulanzen. Die meisten Leitlinien empfehlen einen Medikamentenentzug, der in der Vergangenheit meistens stationär durchgeführt wurde. Ein Paradigmenwechsel führte jetzt dazu, dass die meisten Patienten heute ambulant oder tagesklinisch entzogen werden. Die langfristige Wirksamkeit der beiden Methoden wurde in der vorliegenden Studie untersucht.

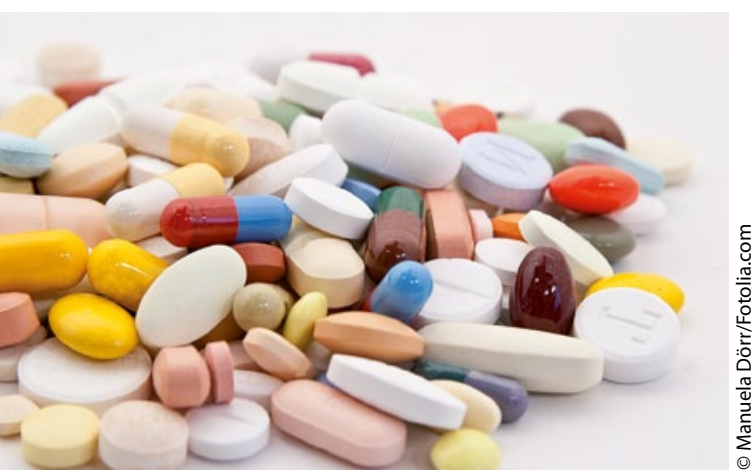

Was tun, wenn die Tabletten, die die Schmerzen bekämpfen sollen, selbst zu Schmerzen führen?

$\mathrm{D}$ efinitionsgemäß handelt es sich bei „Kopfschmerz bei Medikamentenübergebrauch" um chronische Kopfschmerzen bei gleichzeitiger Einnahme von einfachen Analgetika an mehr als 15 Tagen im Monat oder anderen Akutmedikamenten gegen Kopfschmerzen an mehr als zehn Tagen im Monat. In der vorliegenden offenen randomisierten Studie verglichen französische Wissenschaft- ler den ambulanten Entzug mit einer stationären Entzugsbehandlung.

Von den 82 Patienten erhielten in den Jahren 2003 bis 200536 Patienten eine ambulante Entzugsbehandlung und 35 eine stationäre. Untersucht wurden sie nach zwei Monaten und nach zwei Jahren, wobei nach zwei Jahren noch Daten von 34 beziehungsweise 32 Patienten verfügbar waren. In beiden Entzugsverfahren wurden die Medikamente abrupt abgesetzt und Entzugserscheinungen mit Amitriptylin behandelt. Außerdem erhielten die Patienten Metoclopramid gegen Übelkeit und bei Bedarf nicht steroidale Antirheumatika. Der primäre Endpunkt war die Zahl der Kopfschmerztage pro Monat nach zwei Monaten und nach zwei Jahren verglichen mit den Ausgangsdaten. Als Responder wurden Patienten definiert, bei denen der Kopfschmerz wieder an weniger als 15 Tagen im Monat bestand und bei denen an weniger als zehn Tagen im Monat Medikamente zur Akuttherapie eingenommen wurden. Bezüglich der Endpunkte ergab sich zwischen den beiden Behandlungsgruppen kein Unterschied. Die Responderrate betrug jeweils $44 \%$.

Créac'h D et al. In-patient versus out-patient withdrawal programmes for medication overuse headache: a 2-year randomized trial. Cephalalgia. 2011;31(3):1189-98.

Kommentar von Prof. Stefan Evers: Diese Studie reiht sich in mehrere randomisierte Studien ein, die in den letzten Jahren zur Frage des besten Medikamentenentzugs bei dieser Kopfschmerzform vorgelegt wurden. Die Grundsatzfrage dabei ist immer, ob man diese Patienten stationär oder ambulant entziehen beziehungsweise wann man einen stationären Entzug durchführen soll. Die Studien, einschließlich der hier referierten, zeigen konsistent und eindrucksvoll, dass Patienten mit chronischen Kopfschmerzen und Medikamentenübergebrauch genauso gut ambulant oder tagesklinisch entzogen werden können wie stationär. In die klinische Praxis umgesetzt bedeutet dies, dass die einzigen Patienten, bei denen noch über einen stationären Medikamentenentzug nachgedacht werden sollte, diejenigen mit einem Missbrauch von Opioiden oder Benzodiazepinen sind.

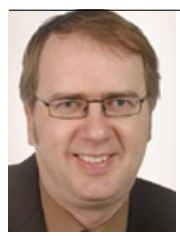

Prof. Dr. med. Stefan Evers Klinik und Poliklinik für Neurologie am Universitätsklinikum Münster

\section{Die Lebensqualität von ambulant oder stationär betreuten Palliativpatienten auf dem Prüfstand}

Die Lebensqualität von Palliativpatienten wird bestimmt durch die körperlichen und psychischen Belastungen ihrer Erkrankung, die familiäre Unterstützung und nicht zuletzt durch ihr spirituelles Erleben. Jetzt wurde untersucht, ob sich das Befinden der Patienten in stationärer und ambulanter Versorgung unterscheidet.

$\mathrm{M}$ aximiliane Jansky und Kollegen gingen retrospektiv der Frage nach, wie Palliativpatienten zu Anfang und Ende einer Behandlung ihr Befinden beurteilten, welche Symptome diese Einschätzung beeinflussten, und ob sich diese zwischen Patienten mit stationärer und ambulanter Versorgung unterscheiden. Anhand von Daten aus der Hospiz- und Palliativerfassung (HOPE) analysierten die Forscher Symptome und Befinden von insgesamt 2.030 Patienten (1.616 stationär, 414 ambulant). Basis waren der Patientenfragebogen (Minimales Dokumen- tationssystems, MIDOS) und der Behandlerfragebogen (Basisbogen). Berücksichtigt wurden Datensätze von Patienten aus den Jahren 2007-2009, für die mindestens zwei vollständige Basisbögen und ein MIDOS-Bogen vorlag. Über $90 \% \mathrm{der} \mathrm{Pa}$ tienten litten an einem Malignom. 53,4\% der Patienten waren weiblich, das mittlere Alter in der Studie betrug 66,9 Jahre. Die ambulanten Patienten verbrachten durchschnittlich mehr Zeit in der Betreuung und waren etwas älter als die stationär betreuten Patienten (68 vs. 66,5 Jahre). 